\title{
Correspondence
}

\section{ESRO X-ray Astronomy Satellite}

Sir,-I would like to comment on the proposal by the ESRO Mission Definition Group for a Lunar Occultation Satellite for high resolution studies of cosmic X-ray sources (Nature, 228, 756; 1970). The authors imply that in order to determine positions of $\mathrm{X}$-ray sources to arc seconds it is necessary to achieve inertial stability of the spacecraft to are seconds. However, this is not the case; in fact, the positional accuracy achieved is typically a small fraction of the vehicle stability. As an example, for Sco $\mathrm{X}-1$ an arc minute position was obtained from a sounding rocket whose pointing accuracy and stability were measured in degrees.

Formally the requirement to achieve high angular resolution is that one be able to reconstruct the inertial attitude of the vehicle on the same angular scale as desired for the X-ray observations. As an example, on NASA's HEAO, which will be rotating at a rate of 30 arc minutes per second, we expect to be able to determine instantaneous orientation to several are seconds and locate X-ray sources to the same precision (Nature, 228, $603 ; 1970$ ).

There is a second requirement which has to do with the effective observing time per source; namely, that the vehicle pointing accuracy be sufficient so that the source can be acquired within the field of view of the X-ray instrument. This is readily achieved. Modulation collimators can be made to have fields of view of many degrees, and even focusing $\mathrm{X}$-ray telescopes, unless they are designed to function at energies in excess of several $\mathrm{keV}$, can have fields of view of a degree or more.

The techniques for accomplishing high angular resolution X-ray measurements are neither expensive nor complex, the principal requirements being large apertures and time resolution. The same considerations apply to high spectral resolution measurements as well.

\section{Yours faithfully,}

\section{HERBERT GURSKY}

Director, Space Research Division,

American Science and Engineering,

11 Carleton Street,

Cambridge, Massachusetts 02142.

\section{Nocturnal Songbird Migration}

Sir,-In their recent communication (Nature, 228, 476; 1970) Gauthreaux and Able have reopened the question of whether passerine nocturnal migrants compensate for wind drift. They have shown that, in the southern United States, on a selection of seven nights in spring and five in autumn, birds flew almost exactly down-wind. Furthermore, on two nights when wind direction changed by about $70^{\circ}$, the migrants' flight directions also changed, so that they still flew down-wind. They propose that radar evidence ${ }^{1}$ (including my own), which disagrees with the hypothesis that birds are drifted from a constant heading, refers not to songbirds but to shorebirds and/or waterfowl.

In England, there is no question of confusion between the radar echoes from small passerine migrants and those from waders and waterfowl. The former fly slowly (about 20 knots) and depart some 40 minutes after dark from the whole land-mass; the latter fly much faster (at least $30 \mathrm{knots}$ ) and depart 1-2 $\mathrm{h}$ before dark from discrete localities, usually on the sea-coast. If our inter- pretation of the radar evidence is incorrect, it is not because we have misidentified the major groups of bird species concerned.

Regrettably, Gauthreaux and Able's presentation does nothing to resolve the question of whether birds fly on chosen tracks or chosen headings. In the situation they have described - that of down-wind flight-these possibilities cannot be distinguished. Furthermore, it would have been useful to know what their migrants did on nights other than the twelve they selected. From my own data, it would also be quite easy to select twelve nights on which the wind coincided in directions with the migrants' tracks; but on many other nights the migrants' tracks followed similar directions even though the wind was partly cross or opposed to these. It was, in part, on this evidence that I concluded that migrants were not drifted from a fixed heading.

Finally, I remain to be convinced that the two instances of change in mean migration direction during the night with change in wind direction disprove the hypothesis that passerine migrants usually compensate for wind drift. Firstly, different birds are involved at different times of night. Might they not be different species with different preferred tracks? Secondly, we are not told exactly where the observations were made. If the spring observations refer to Lake Charles, Louisiana, then birds departing from the coast of the Gulf of Mexico at dusk and flying NE (some passing the observation point at $02.40 \mathrm{GMT}$ ) would all have passed well before the second set of observations were made at $06.20 \mathrm{GMT}$; so it may not be surprising that a different mean flight direction. was seen at the later hour as it could have involved a very different group of migrants.

\section{Yours faithfully,}

\section{P. R. Evans}

Department of Zoology,

University of Durham,

South Road,

Durham.

${ }^{1}$ Nisbet, I. C. T., and Drury, W. H., Bird Banding, 38, 173 (1967).

2 Belirose, F. C., Proc. Fourteenth Intern. Ornithol. Cong., 281 (1967).

s Evans, P. R., J. Zool., 150, 319 (1966).

- Lack, D., Ibis, 111, 253 (1969).

\section{Biochemical Theology'}

Sir,-

I have isolated from murine

Urine,

Collected from the Church or House

Mouse,

A substance which induces, by injection,

Reflexion,

Verbalization

And prognostication

(Sagacious

And, of course, efficacious).

Is this what makes Dr Crick

Tick?

If not,

What?

Yours faithfully,

Trinity College,

A. G. Ogston

Oxford.

2 Crick, F., Nature, 228, 613 (1970). 